\title{
Circulating and circular RNAs and the need for rationalization and synthesis of the research spiral
}

\author{
Pavel Dvorak ${ }^{1,3, D-F}$, Sarah Leupen ${ }^{2, D-F}$, Pavel Soucek ${ }^{3, D-F}$ \\ ${ }^{1}$ Department of Biology, Faculty of Medicine in Pilsen, Charles University, Czech Republic \\ ${ }^{2}$ Department of Biological Sciences, University of Maryland, Baltimore, USA \\ ${ }^{3}$ Biomedical Center, Faculty of Medicine in Pilsen, Charles University, Czech Republic \\ A - research concept and design; $B$ - collection and/or assembly of data; $C$ - data analysis and interpretation; \\ $\mathrm{D}$ - writing the article; $\mathrm{E}$ - critical revision of the article; $\mathrm{F}$ - final approval of the article
}

\section{Address for correspondence}

Pavel Dvorak

E-mail: Pavel.Dvorak@lff.cuni.cz

\section{Funding sources}

This work was supported with the National

Sustainability Program I (NPU I) provided

by the Ministry of Education, Youth and Sports

of the Czech Republic (project No. L01503);

and Charles University Research Center program

UNCE/MED/006 "University Center of Clinical

and Experimental Liver Surgery".

Conflict of interest

None declared

Received on November 11, 2017

Reviewed on February 28, 2018

Accepted on August 9, 2018

Published online on March 6, 2019

\begin{abstract}
In this essay, we aim to draw a short comparison between 2 important research topics - circular and circulating RNAs - and show how they are connected. The findings described here in the field of circular RNAs, which are still quite obscured by the rapidly expanding body of knowledge in biology, have added another dimension to our view of the process of gene expression, which is formed by a more complex network of molecule interactions than we previously thought. The term "circulating RNAs" refers to a broad spectrum of RNA fragments originating from different sources, such as physiologically dying cells, sites of inflammation or cancer cells, and fragments floating in human liquid tissues together with other elements. Fragments of nucleic acids circulating in blood are emerging as promising biomarkers in different medical conditions. Interestingly, circular RNAs have been found to be present in human blood and form a fraction of circulating RNAs. In addition to updating readers on these fast-developing areas of biology, we also stress the need for the study of complex networks of molecule interactions as whole structures (in unison with the thoughts of systems biology), as opposed to the trend toward searching for individual key player molecules. Fundamentally, we want to add to the rationalization and synthesis of new research findings in the scientific literature, because this direction is important not only for students, teachers and researchers, but also for the general population.
\end{abstract}

Key words: synthesis, circular RNA, circulating RNA, complex networks, systems biology

Cite as

Dvorak P, Leupen S, Soucek P. Circulating and circular RNAs and the need for rationalization and synthesis of the research spiral. Adv Clin Exp Med. 2019;28(6):833-838. doi:10.17219/ acem/94148

DOI

10.17219/acem/94148

\section{Copyright}

Copyright by Author(s)

This is an article distributed under the terms of the

Creative Commons Attribution Non-Commercial License

(http://creativecommons.org/licenses/by-nc-nd/4.0/) 


\section{Introduction}

The discovery of the unexpected amount and roles of circular RNA molecules in human cells is a recent example of how new research findings can be concealed in the stream of the rapidly expanding body of scientific knowledge in biology. Although articles focusing on the theme of circular RNAs have been spreading round the world for about 5 years, information about this phenomenon is still quite unknown among non-specialists, and could even be confused with the topic of circulating RNAs, another widely researched topic with a similar name. The findings in the field of circular RNAs described in this article have added another dimension to our view of the process of gene expression. Similarly, the recent advances in the field of circulating nucleic acids and their clinical applications are very surprising.

It will probably take some more time for these discoveries to be suitably incorporated into university curricula, so we hope that this article can "fill in the gap" at this time. Although part of our goal is to help readers understand the distinctions between these topics in biological research, as well as the importance of each topic, we also aim to demonstrate some connections between them. Additionally, we wish to stress the need for the study of these highly complex networks of molecule interactions as whole structures in opposition to the recently prevalent search for individual key player molecules. Broadly, we want to promote the rationalization and synthesis of new research findings in the scientific literature in general, which is important not only for students, teachers and researchers, but also for the general population. Notably, we are aware that the whole theme of circulating cells and molecules, derived from different sources of tissues and cells, in human peripheral blood is much broader than discussed here, and we leave untouched several broad topics such as circulating nucleosomes and circulating tumor cells.

\section{Circular RNAs}

The view that the dominant RNA isoforms produced from eukaryotic genes were unstable variants of messenger RNA (mRNA) has considerably changed after the discovery of the high number of circular RNAs (circRNAs) widely present in human cells. ${ }^{1-3}$ Circular RNAs have broadened the spectrum of long non-coding RNAs (lncRNAs), which were arbitrarily defined as having more than 200 nucleotides. LncRNAs may be standalone transcription units, or they may be transcribed from enhancers, promoters or introns of other genes; from pseudogenes; or antisense to other genes with varying degrees of overlap. Some members of lncRNAs have been shown to affect regulation of gene expression at the transcriptional as well as at post-transcriptional level. ${ }^{4,5}$ Generally, the 3' and 5' ends of circRNAs are covalently linked during the process named "backsplicing" or "head-to-tail splicing", an alternative splicing of precursor mRNAs (pre-mRNAs) in which a downstream splice donor is joined to an upstream splice acceptor (in the reverse orientation to linear splicing; demonstrated in Fig. 1). 6,7

Well-known genes from several species such as the mouse sex determining region of Chr Y (Sry), rat sex hormone binding globulin $(S h b g)$, monkey solute carrier family 8 member A1 (SLC8A1, also known as Ncx1) or human ETS proto-oncogene 1, transcription factor (ETS1) and cytochrome P450 family 2 subfamily C member 18 (CYP2C18) were associated with the first reported examples of spliced circRNAs. However, these "strange" molecules were soon

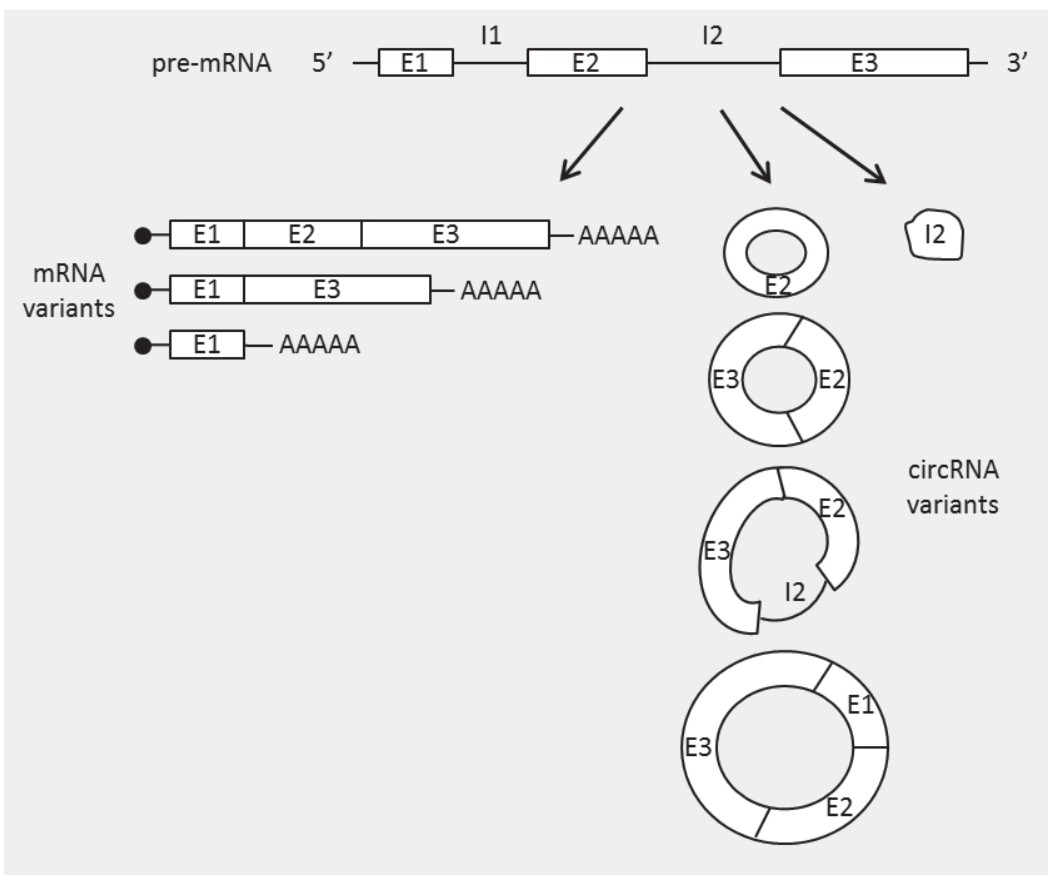

Fig. 1. Biogenesis of circular RNAs. Splicing variants of mRNA are generated in the process of canonical (linear) splicing, and circular RNA (circRNA) variants originate through the process called backsplicing or from intronic transcripts

E-exon; I - intron. 
found to be unexpectedly more widespread, following a steep increase of information about these molecules coming from next-generation sequencing studies, which attempted to find genomic rearrangements in cancer samples. ${ }^{6}$ The general key findings of several subsequent studies were that circRNAs were localized to the cytoplasm, expressed in high amounts in thousands of human genes, exhibited cell type-specific expression, demonstrated conservation between mouse and human, and were remarkably stable, most likely due to their circular structure. ${ }^{1-3,8}$ A great diversity of circRNA isoforms can be generated from a single genomic locus and can consist of 1 or more exons and even contain unspliced intronic sequences. ${ }^{6,7}$ Furthermore, circRNAs derived from antisense, 5' or 3' untranslated or intergenic genomic regions were reported.

What do we know about the functional significance of these molecules? To date, several studies have shown that circRNAs can be involved in RNA interference pathways, suggesting that the more stable circRNAs compete with messenger RNAs (mRNAs) for microRNA (miRNA) binding in the cytoplasm and consequently interfere in gene regulation. One of the most studied examples is human ciRS-7 (also known as CDR1as), which is transcribed in the antisense orientation from the cerebellum degeneration-related antigen $1(C D R 1)$ gene. Its overexpression acts as a miR-7 sponge, arresting this miRNA and therefore elevating the level of its targets. MiR-7 is known to regulate, among others, epidermal growth factor receptor (EGFR) expression. The EGFR is a cell surface protein (tyrosine kinase) that binds to epidermal growth factors and controls cell growth and proliferation. Mutations in the EGFR gene have been associated with the development of several types of cancers, especially lung cancer. In a similar manner, mouse Sry circRNA harbors 16 putative target sites for miR-138 and its ability to diminish the silencing activity of this miRNA has been observed in several studies. ${ }^{3,9-11}$ In humans, miR-138 can interact with several transcripts related to cholangiocarcinoma, colorectal, ovarian and lung cancer, and also hematopoietic tumors such as lymphocytic leukemia. ${ }^{9}$

Furthermore, the power of circRNAs to act as sponges for other molecules has been extended to proteins (e.g., DNA-/RNA-binding proteins), thus establishing a more general phenomenon. With regard to this aspect, the circRNA that is derived from the $m b l$ locus in Drosophila (circMbl) harbors binding sites for the MBL protein itself.,12 Interestingly, MBL is able to induce circMbl production. Generation of this circRNA consequently renders the pre-mRNA non-productive, limiting further production of MBL protein. CircMbl tethers and decoys MBL, which prevents further generation of circMbl and instead reactivates productive $m b l \mathrm{mRNA}$ production. Therefore, circMbl seems to be an intricate entity of an MBL autoregulatory circuit. Furthermore, circMbl also encompasses highly conserved miRNA binding sites and therefore circMbl could in fact have multi-faceted roles in the Drosophila brain. ${ }^{7,12}$
Different from most circRNAs, circRNAs with intron sequences are usually resident in the nucleus, which is similar to the observations of the nuclear restriction of linear RNAs containing retained introns. It has been reported that nuclear circRNAs can bind to the eukaryotic RNA polymerase II to regulate gene transcription. ${ }^{13}$ For instance, ciankrd52, which originates from the $2^{\text {nd }}$ intron region of the ankyrin repeat domain 52 (ANKRD52) gene, can interact with the elongating polymerase II complex and accumulates at transcription sites, which promotes transcription of its parental gene ANKRD52. Two other nuclear circRNAs, circ EIF3J and circ PAIP2, which contain one potential U1 snRNA-binding site in their retained introns, interact with the U1 snRNP and promote the transcription of their parental genes, EIF3J and PAIP2, suggesting that nuclear circRNAs, U1 snRNP, and polymerase II might interact with each other at promoter regions. ${ }^{13}$

CircRNAs that harbor binding sites for enzymes and their substrates are likely to function as scaffolds facilitating co-localization and reaction kinetics. This is perhaps best exemplified by circFOXO3, which is highly expressed in non-cancer cells and associated with cell proliferation, apoptosis and cell cycle. CircFOXO3 regulates the cell cycle by combining with CDK2 and CDKN1A (previously p21), which hinders the combination between CDK2 and cyclin E. Meanwhile, circFOXO3 decreases TP53 (p53) expression and increases FOXO3 (Foxo3) expression, which contributes to cell apoptosis and inhibition of proliferation. ${ }^{14,15}$

CircBase (circbase.org), one of the several freely available databases where public data sets of circRNAs can be accessed, downloaded and browsed within the genomic context, lists tens of thousands of circRNAs discovered in many different human cells. ${ }^{16}$ As well as being an informational resource, the site provides custom scripts in Python that can locate circRNA sequences in any RNA sequence data, so researchers and students can use this site not just to learn more about circRNAs but also to discover new ones.

Recently, circRNAs were located not just inside cells, but also in the bloodstream. Using RNA sequencing of clinical samples, Memczak et al. found around 2,400 circRNA candidates expressed in human whole blood and, moreover, observed that the overall circRNA expression level in blood is unexpectedly similar to that of neuronal tissues, where circRNAs are highly abundant. ${ }^{17}$ Thus, some circular RNAs are also circulating RNAs. This leads us directly to our $2^{\text {nd }}$ theme - circulating RNAs and their potential for liquid biopsy.

\section{Circulating RNAs and liquid biopsy}

The presence of cell-free DNA (cfDNA) in human peripheral blood is a well-established phenomenon, first reported by Mandel and Metais in $1948 .{ }^{18}$ Fragments of DNA 
(double-stranded, mostly approx. $150 \mathrm{bp}$ in length) are shed into the bloodstream from dying cells during cellular turnover or other forms of cell death; alternatively, they can be actively secreted from cells in association with membranederived vesicles or conjugated with lipid-protein complexes (both mechanisms are also valid in the case of tumor cells). Researchers working in this field proposed the possibility of non-invasive testing for blood-based cfDNA in different clinical circumstances and, especially in cases of prenatal (e.g., non-invasive prenatal testing - NIPT) and cancer (e.g., detection of EGFR mutations from the plasma of lung cancer patients) testing, this approach has achieved considerable success. ${ }^{19-21}$

In a manner similar to cfDNA, fragments of RNAs are released into the blood by several microvesicle-dependent or microvesicle-independent mechanisms. Although cellfree RNA (cfRNA) is generally even less stable in blood circulation than cfDNA, research has shown that different kinds of vesicles (exosomes, microvesicles or apoptotic bodies) can sequester fragments of mRNA, short and long non-coding RNAs as well as other biomolecules. A large variety of cell types can release extracellular vesicles and it was discovered that miRNA within circulating exosomes can be biologically active at distant sites. ${ }^{22}$

During carcinogenesis, this process of encapsulation can result in the formation of so-called tumor-educated platelets (TEPs). ${ }^{21}$ Tumor-educated platelets have emerged as important players in different tumor-related processes including invasion and establishment of distant metastasis. Alternatively, cfRNA can be conjugated with RNA-binding proteins such as Argonaut 1 and 2.

Thus, a so-called "liquid biopsy" could provide a promising alternative or complement to tissue biopsy, which is usually performed on tissue taken from the primary tumor, if it is accessible. Furthermore, tissue biopsy is limited in that it reflects the molecular composition of only a small fraction of tumor cells at the time of sample-taking. Progress in technology, specifically the advent of massively parallel sequencing (next-generation sequencing - NGS), has provided unprecedented opportunities to investigate cell-free nucleic acids in a genome-wide fashion and at single-base resolution with the possibility to detect the wider intra-patient tumor heterogeneity. ${ }^{23}$ Moreover, cell-free nucleic-acid-based profiling of cancer patients offers a number of critical advantages for essentially realtime monitoring of tumor response to therapy in cancer patients. Krug et al. investigated whether using a combined isolation of exosomal RNA and cfDNA could improve blood-based liquid biopsy for EGFR mutation detection in non-small-cell lung cancer (NSCLC) patients. ${ }^{24}$ They concluded that combining exosomal RNA and ctDNA increased the sensitivity for EGFR mutation detection in plasma, with the largest improvement seen in the subgroup of M0/M1a disease patients known to have low levels of ctDNA, and posed challenges for mutation detection on ctDNA alone.

\section{Challenges in detecting circular RNAs and circulating transcriptome}

We can stress several issues among the challenges for circRNA detection in RNA sequencing data. Firstly, variations in preparation protocols alter the amount of circRNA in a library depending on the RNA purification method, size selection and oligo(dT) vs random priming. Secondly, there are several known sources of artefacts from common RNA-sequencing protocols, e.g., reverse transcriptase can join 2 distinct RNA molecules in a non-canonical order, particularly when the 2 RNAs contain a common sequence; 2 distinct cDNAs may be ligated together in non-canonical order during adaptor ligation; reverse transcriptase can displace cDNA from the template, generating a single cDNA that contains multiple copies of a circRNA. Thirdly, a convolution of homology and sequencing errors can lead to false alignments to a backsplice junction. ${ }^{25}$

It should be noted that the vast majority of research in the circulating transcriptome field are single-centered retrospective studies and that the cohorts typically used are insufficiently powered. As a consequence, there are many non-overlapping and even contradictory reports relating to the circulating transcriptome. These differences are primarily because of biological and technical variation between studies such as the starting material used in experiments (e.g., purification of cells, cell types, control populations used), technological platforms (e.g., microarray, quantitative real-time polymerase chain reaction (qRT-PCR), next-generation sequencing), and differing statistical methodologies used. Such confounding factors are especially problematic for studies of the circulating transcriptome which are characterized by low-quality and low-quantity RNA. ${ }^{26,27}$

At present, the low amount and high degradation rate of tumor-derived cfRNAs (with the exception of miRNAs and circRNAs) together with a high admixture of their physiological counterparts, derived from non-neoplastic cells, still pose major challenges for the development of sensitive and robust detection methods, which would be highly valuable in routine oncology practice. Importantly, due to their increased stability relative to other RNA fragments in the open blood environment and also their tissue specificity, circulating miRNAs have captured the attention of many researchers and are now being extensively studied as attractive new biomarkers across different medical fields. ${ }^{26,27}$ Circular RNAs (circRNAs) have also been shown to be more stable than their linear counterparts, ${ }^{28}$ and after the discovery that circular RNAs can also circulate in the human bloodstream they quickly joined miRNAs at the top of biomarker research. ${ }^{11}$ For example, as human brain tissue is mostly inaccessible in patients with neurological disorders, peripheral blood presents a primary source of tissue for biomarker-based diagnostics. 


\section{Rationalization and synthesis of new research findings}

It has become clear that the wide spectrum of noncoding RNAs can interact with each other and regulate the amount of coding RNAs and, in combination with transcription factors and other regulative elements, form a highly complex network of molecule interactions that results in time- and site-specific gene expression (shown in a simplified form in Fig. 2). Similarly, complex networks have also been revealed in other physiological processes such as metabolic and signaling pathways. Because the evolution of complex networks of interactions seems to be a natural tendency, many other examples on a cellular level are likely to be uncovered.

The increasing amount of information about the individual components of the complex networks of molecule interactions, coming from high-throughput "-omics" techniques, allows us to raise questions which would fit into the field covered by systems biology. ${ }^{29-31}$ Examples of such questions are as follows: "Can we characterize the complex network of molecule interactions as a whole structure without just summarizing the characteristics of its individual components?"; "Does this higher level of network characteristics show some patterns which are shared by other complex networks consisting of different compounds?"; "Can some kind of classification of complex networks be defined based on such patterns?"; "Can normal and pathological states of complex networks be distinguished according to such patterns?"; "Can we foresee how a change in a component would change the behavior of the whole complex network?"; "How many individual components of a complex network need to be disrupted before an impact on the whole structure can be detected?"; "Would a treatment strategy taking into account system biology results be more successful than a strategy focusing only on a key component of the complex network?".

Researchers try to find answers to such questions through the development of different network diagrams and computational and mathematical models. It is a rapidly developing area of biology research, however, it could be challenging to find the reasonable level of these models which would be comprehensible for a broader biology-educated community or even broader population. Open source software platforms and databases such as STRING (https:// string-db.org/) and Cytoscape (http://www.cytoscape.org/) may be employed for visualizing complex networks and integrating these with biological data of different kinds.

\section{Conclusions and perspectives}

The discoveries that circRNAs are abundantly expressed in human cells and are involved in gene expression regulation through regulatory elements such as miRNAs and RNA-binding proteins have significantly broadened our view of the whole process of gene expression. These molecules have emerged as attractive targets for primary as well as applied research and a lot of their characteristics were revealed. ${ }^{11,32}$ However, although the initiation of eukaryotic translation relies on the cap structure in the 5' end of mRNA, protein-coding circRNAs, circZNF609

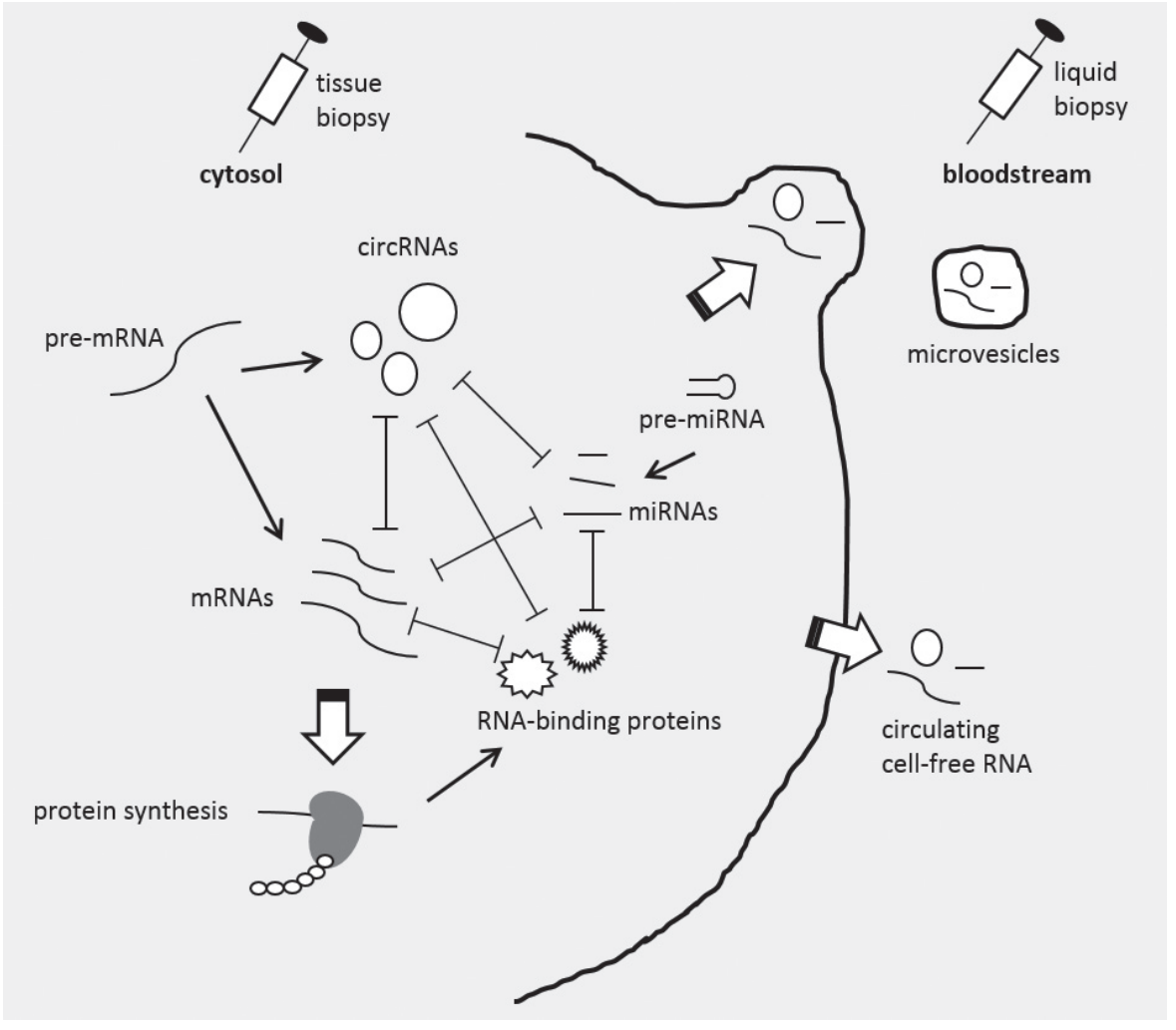

Fig. 2. Tissue vs liquid biopsy in the context of the complex network of molecule interactions involved in protein synthesis regulation 
in human muscle cells and circMbl in fruit fly head have been described very recently and have opened a completely new field within this topic. ${ }^{33,34}$

RNA fragments originating from different sources are floating in human liquid tissues and are called circulating RNAs; if not encapsulated, they are degraded in a short period of time. Interestingly, circRNAs have been found to circulate in human blood and form a fraction of circulating RNAs. Because circRNAs are reasonably stable, many research teams have started to evaluate them as prospective biomarkers. ${ }^{32,35}$ These recent findings have created an intersection between the 2 otherwise different topics - circular and circulating RNAs.

One of the main goals of current cancer research is to find the key players on the molecular level and through their modification open the possibility of reliable interventions in clinical situations. However, in the context of complex networks of molecule interactions, it is questionable if this approach alone will lead to useful solutions. Research should also focus on these complex networks of molecular interactions as whole structures, e.g., by looking for characteristic expression profiles across different types of molecules and examining how these profiles differ in physiological and pathophysiological states. We would like to encourage this type of research as well as education focused on the rationalization and synthesis of new research findings and their comparison in the context of other highly complex subsystems present in living organisms.

\section{References}

1. Jeck W, Sorrentino J, Wang K, et al. Circular RNAs are abundant, conserved, and associated with ALU repeats. RNA. 2012;19(2):141-157. doi:10.1261/rna.035667.112

2. Salzman J, Gawad C, Wang P, Lacayo N, Brown P. Circular RNAs are the predominant transcript isoform from hundreds of human genes in diverse cell types. PLoS One. 2012;7(2):e30733. doi:10.1371/journal. pone.0030733

3. Memczak S, Jens M, Elefsinioti A, et al. Circular RNAs are a large class of animal RNAs with regulatory potency. Nature. 2013;495(7441): 333-338. doi:10.1038/nature11928

4. Fatica A, Bozzoni I. Long non-coding RNAs: New players in cell differentiation and development. Nat Rev Genet. 2013;15(1):7-21. doi:10. $1038 /$ nrg3606

5. Kung J, Colognori D, Lee J. Long noncoding RNAs: Past, present, and future. Genetics. 2013;193(3):651-669. doi:10.1534/genetics.112.146704

6. Barrett S, Salzman J. Circular RNAs: Analysis, expression and potential functions. Development. 2016;143(11):1838-1847. doi:10.1242/dev.128074

7. Ebbesen K, Kjems J, Hansen T. Circular RNAs: Identification, biogenesis and function. Biochim Biophys Acta. 2016;1859(1):163-168. doi:10. 1016/j.bbagrm.2015.07.007

8. Salzman J, Chen R, Olsen M, Wang P, Brown P. Cell-type specific features of circular RNA expression. PLoS Genet. 2013;9(9):e1003777. doi:10.1371/journal.pgen.1003777

9. Kulcheski F, Christoff A, Margis R. Circular RNAs are miRNA sponges and can be used as a new class of biomarker. J Biotechnol. 2016;238: 42-51. doi:10.1016/j.jbiotec.2016.09.011

10. Hansen $T$, Jensen $T$, Clausen $B$, et al. Natural RNA circles function as efficient microRNA sponges. Nature. 2013;495(7441):384-388. doi:10.1038/nature11993

11. Huang S, Yang B, Chen B, et al. The emerging role of circular RNAs in transcriptome regulation. Genomics. 2017;109(5-6):401-407. doi:10. 1016/j.ygeno.2017.06.005
12. Ashwal-Fluss $\mathrm{R}$, Meyer $\mathrm{M}$, Pamudurti $\mathrm{N}$, et al. circRNA biogenesis competes with pre-mRNA splicing. Mol Cell. 2014;56(1):55-66. doi:10. 1016/j.molcel.2014.08.019

13. Huang G, Li S, Yang N, Zou Y, Zheng D, Xiao T. Recent progress in circuIar RNAs in human cancers. Cancer Lett. 2017;404:8-18. doi:10.1016/j. canlet.2017.07.002

14. Kristensen L, Hansen T, Venø M, Kjems J. Circular RNAs in cancer: Opportunities and challenges in the field. Oncogene. 2017;37(5):555-565. doi:10.1038/onc.2017.361

15. Zhang H, Jiang L, Sun D, Hou J, Ji Z. CircRNA: A novel type of biomarker for cancer. Breast Cancer. 2017;25(1):1-7. doi:10.1007/s12282017-0793-9

16. Glažar P, Papavasileiou P, Rajewsky N. circBase: A database for circular RNAs. RNA. 2014;20(11):1666-1670. doi:10.1261/rna.043687.113

17. Memczak S, Papavasileiou P, Peters O, Rajewsky N. Identification and characterization of circular RNAs as a new class of putative biomarkers in human blood. PLoS One. 2015;10(10):e0141214. doi:10.1371/ journal.pone.0141214

18. Mandel $P$, Metais $P$. Les acides nucléiques du plasma sanguin chez I'homme. C R Seances Soc Biol Fil. 1948;142(3-4):241-243.

19. Vermeesch J, Voet T, Devriendt K. Prenatal and pre-implantation genetic diagnosis. Nat Rev Genet. 2016;17(10):643-656. doi:10.1038/ nrg.2016.97

20. Volik S, Alcaide M, Morin R, Collins C. Cell-free DNA (cfDNA): Clinical significance and utility in cancer shaped by emerging technologies. $\mathrm{Mol}$ Cancer Res. 2016;14(10):898-908. doi:10.1158/1541-7786.mcr-16-0044

21. Sorber L, Zwaenepoel K, Deschoolmeester V, et al. Circulating cellfree nucleic acids and platelets as a liquid biopsy in the provision of personalized therapy for lung cancer patients. Lung Cancer. 2017; 107:100-107. doi:10.1016/j.lungcan.2016.04.026

22. Falcon-Perez J, Royo F. Circulating RNA: Looking at the liver through a frosted glass. Biomarkers. 2015;20(6-7):339-354. doi:10.3109/13547 50x.2015.1101785

23. Jiang $P$, Lo Y. The long and short of circulating cell-free DNA and the ins and outs of molecular diagnostics. Trends Genet. 2016;32(6):360-371. doi:10.1016/j.tig.2016.03.009

24. Krug A, Enderle D, Karlovich C, et al. Improved EGFR mutation detection using combined exosomal RNA and circulating tumor DNA in NSCLC patient plasma. Ann Oncol. 2018;29(3):700-706. doi:10.1093/ annonc/mdx765

25. Szabo L, Salzman J. Detecting circular RNAs: Bioinformatic and experimental challenges. Nat Rev Genet. 2016;17(11):679-692. doi:10.1038/ nrg. 2016.114

26. Fernandez-Mercado M, Manterola L, Larrea $E$, et al. The circulating transcriptome as a source of non-invasive cancer biomarkers: Concepts and controversies of non-coding and coding RNA in body fluids. J Cell Mol Med. 2015;19(10):2307-2323. doi:10.1111/jcmm.12625

27. Tiberio P, Callari M, Angeloni V, Daidone M, Appierto V. Challenges in using circulating miRNAs as cancer biomarkers. Biomed Res Int. 2015;2015:1-10. doi:10.1155/2015/731479

28. Enuka Y, Lauriola M, Feldman M, Sas-Chen A, Ulitsky I, Yarden Y. Circular RNAs are long-lived and display only minimal early alterations in response to a growth factor. Nucleic Acids Res. 2015;44(3):13701383. doi:10.1093/nar/gkv1367

29. Breitling R. What is systems biology? Front Physiol. 2010;1:9. doi:10. 3389/fphys.2010.00009

30. Kitano H. Systems biology: A brief overview. Science. 2002;295(5560): 1662-1664. doi:10.1126/science.1069492

31. Yurkovich J, Palsson B. Quantitative-omic data empowers bottom-up systems biology. Curr Opin Biotechnol. 2018;51:130-136. doi:10.1016/j. copbio.2018.01.009

32. Li M, Ding W, Sun T, et al. Biogenesis of circular RNAs and their roles in cardiovascular development and pathology. FEBS J. 2018;285(2): 220-232. doi:10.1111/febs.14191

33. Legnini I, Di Timoteo G, Rossi F, et al. Circ-ZNF609 is a circular RNA that can be translated and functions in myogenesis. Mol Cell. 2017;66(1): 22-37.e9. doi:10.1016/j.molcel.2017.02.017

34. Pamudurti N, Bartok O, Jens M, et al. Translation of circRNAs. Mol Cell. 2017;66(1):9-21.e7. doi:10.1016/j.molcel.2017.02.021

35. Zhang $H$, Jiang L, Sun D, Hou J, Ji Z. CircRNA: A novel type of biomarker for cancer. Breast Cancer. 2018;25(1):1-7. doi:10.1007/s12282017-0793-9 